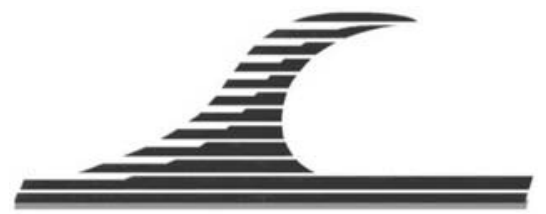

Mots-clés : Couverture sédimentaire, Sable, Vase, Distribution, Bathymétrie, Lagune Adjin, Côte d'Ivoire

(C) Editions Paralia CFL

\title{
Analyse granulométrique et environnement de dépôts des sables superficiels de la lagune Adjin (Côte d'Ivoire)
}

\author{
Yao Alexis N'GUESSAN ${ }^{1}$, Valérie WOGNIN ${ }^{1}$, Aoua COULIBALY ${ }^{1}$, \\ Sylvain MONDE ${ }^{1}$, Ted Edgard WANGO ${ }^{1}$, Kouamé AKA ${ }^{1}$
}

1. Université de Cocody, UFR des Sciences de la Terre et des Ressources Minières, Laboratoire de Géologie Marine et de Sédimentologie,

22 BP 582 Abidjan 22, Côte d'Ivoire.

alestouxis@yahoo.fr

\section{Résumé :}

Les analyses granulométriques et les levés bathymétriques effectués en lagune Adjin, ont permis de caractériser les sédiments sableux et la morphologie du fond de cette lagune. Sur le fond de la lagune, les sédiments sont constitués majoritairement de vases localisées dans les fonds lagunaires alors que sur la bordure de la lagune, on rencontre essentiellement des sables. Les galets et les graviers se localisent par endroit, sur le pourtour de la lagune, et à proximité des débouchés des rivières Djibi et Bété. L'essentiel des sables de la lagune Adjin provient d'un environnement de type rivières. La majorité des sables se déplace par saltation. Le mode de transport des sables fins est dominé par le transport par suspension. Les sables moyens se déplacent essentiellement par saltation. Le transport par roulement est le mode de transport dominant des sables grossiers. Cette étude a permis de réaliser les cartes bathymétriques et de répartition des sédiments, ainsi que la détermination de l'environnement de dépôts des sables.

Soumis le 15 septembre 2010, accepté le 27 juin 2011, en ligne le 26 septembre 2011. La seule version examinée est celle écrite en français. La ou les autres versions n'étant pas examinées par le comité de rédaction de la revue, sont donc publiées sous l'entière responsabilité du ou des auteurs.

A TRANSLATED VERSION IN ENGLISH IS AVAILABLE ONLINE

Pour citer cet article :

N'GUESSAN Y.A., WOGNIN V., COULIBALY A., MONDE S., WANGO T.E., AKA K. (2011). Analyse granulométrique et environnement de dépôts des sables superficiels de la lagune Adjin (Côte d'Ivoire). Revue Paralia, Vol. 4, pp 6.1-6.14.

DOI:10.5150/revue-paralia.2011.006 (disponible en ligne - http://www.paralia.fr - available online) 


\section{Introduction}

D'une superficie totale d'environ $1200 \mathrm{~km}^{2}$, le milieu lagunaire de Côte d'Ivoire est situé dans la zone littorale au Sud de la Côte d'Ivoire et forme l'interface entre les milieux continental, fluvial et marin. Il est constitué de trois lagunes principales dont la lagune Ebrié qui est en relation permanente avec la mer par le canal de Vridi (ouvert en 1950) et en période de crue par l'embouchure temporaire du Comoé à Grand-Bassam (fermé depuis 1975). Il est alimenté en eau douce par le fleuve Comoé, le fleuve Agnéby et le fleuve Mé. Les lagunes intérieures Adjin et Potou reliées entre elles par un canal naturel, sont situées sur sa rive Nord et représentent près du dixième de la surface du plan d'eau soit $43 \mathrm{~km}^{2}$ sur $523 \mathrm{~km}^{2}$ (TASTET, 1979).

La lagune Adjin, objet de cette étude est comprise entre les $5^{\circ} 21^{\prime}$ et $5^{\circ} 30^{\prime}$ de latitude Nord et les $3^{\circ} 45^{\prime}$ et $3^{\circ} 57^{\prime}$ de longitude Ouest, avec une superficie de $20,2 \mathrm{~km}^{2}$. Lagune intérieure appartenant au grand système lagunaire Ebrié, elle est alimentée en eau douce par le fleuve Mé et les rivières, Djibi et Bété (voir figure 1). Elle constitue, avec les écosystèmes adjacents, de véritables supports de subsistance pour les populations environnantes. Ces populations installées sur les rives de la lagune ont entre autres, pour activité économique principale la pêche. Ces activités entraînent un changement défavorable des caractéristiques naturelles de ces milieux saumâtres, siège de phénomènes complexes liés aux échanges entre eaux douces et marines.

Cette étude traitera de la granulométrie et de l'environnement de dépôt des sables superficiels de la lagune Adjin. L'analyse granulométrique permettra de mettre en évidence le comportement hydrodynamique des grains de sable en tenant compte à la fois de leur répartition, de leur taille, de leur nature et de leur origine. Ensuite l'étude s'intéressera au mode de transport de ces sables afin de dégager leur environnement de dépôt. Elle se terminera par une comparaison entre les différents paramètres granulométriques des sables par une analyse statistique.


Figure 1. Localisation de la lagune Adjin. 
Analyse granulométrique et environnement de dépôts des sables superficiels de la lagune Adjin (Côte d'Ivoire) : 6.3

\section{Matériel et méthodes}

\subsection{Levés de sondes bathymétriques}

Les levés bathymétriques ont été effectués à partir d'un échosondeur de type Lowrance, modèle LMS-160 et Global Map ${ }^{\mathrm{TM}} 1600$, couplé à un récepteur GPS pour l'acquisition des sondes. Elles ont été mesurées avec une fréquence de $160 \mathrm{kHz}$ qui permet aux signaux émis de ne pas pénétrer les vases (LOWRANCE, 1998) et la lecture faite directement sur l'écran de l'unité centrale. Les corrections sont effectuées afin de déterminer la profondeur réelle à chaque point. Ces corrections concernent la profondeur d'immersion du transducteur et la marée dynamique (ABE, 2005).

Les sondes réelles sont traitées et analysées afin de tracer des contours de cartes, des courbes d'isovaleurs dans un plan de la lagune Adjin.

\subsection{Prélèvements des sédiments superficiels}

L'échantillonnage sédimentologique en lagune Adjin, s'est déroulé à l'aide d'une benne Van Veen. Ces prélèvements ponctuels ont été positionnés par un récepteur GPSGARMIN dont la précision est environ de $2 \mathrm{~m}$ (WOGNIN, 2004).

Pour l'étude granulométrique, les sédiments meubles, après conditionnement (lavage et séparation de la fraction supérieure à $63 \mu \mathrm{m}$; attaques chimiques à l'acide chlorhydrique et à l'eau oxygénée pour l'élimination respective des carbonates et de la matière organique; séchage à l'étuve) ont subi un tamisage sur une colonne de quinze tamis vibrants de la série AFNOR, de mailles comprises entre $63 \mu \mathrm{m}$ et $5 \mathrm{~mm}$ (N'GUESSAN et al., 2008). La cartographie des faciès des sédiments superficiels de la lagune Adjin a été réalisée.

A partir des formules de FOLK et WARD (1957) basées sur l'unité $\Phi$ (où $\Phi=-\log _{2} \mathrm{~d}$, avec $\mathrm{d}$ désignant le diamètre des particules en millimètre), six paramètres de distribution granulométriques ont été calculés. Ce sont : la médiane (Md), le mode (Mo), le grain moyen (M), l'indice de tri (IT), l'écart type ou classement ou Sorting (So) et l'asymétrie ou le Skewness (Sk). Des cartes de répartition des sédiments (galets et graviers, sables, vases) ont été réalisées.

\subsection{Le test de Visher}

Il met en relation la granulométrie et le mode de transport. L'auteur VISHER (1969) porte en ordonnées les pourcentages cumulés et en abscisses la taille (en unité phi $(\Phi)$ ) des classes granulométriques correspondantes. Cette méthode permet de définir trois modes de transport qui sont : la suspension, la saltation et le roulement (ou reptation, ou charriage ou encore "surface creep"). 


\subsection{Détermination des milieux de dépôts}

Les diagrammes Md-So et Sk-Md, utilisés établissent une discrimination entre les sables de plages, de rivières, de dunes côtières et de dunes continentales (BOUMIR, 1985). Le diagramme de dispersion de MOIOLA et WEISER (1968) permet de mettre en relation la granulométrie et l'environnement de dépôt des sédiments. Les paramètres sont calculés à partir d'une progression géométrique des tamis de 1/4 $\Phi$. Cette méthode permet de déduire l'origine des sables (rivières, plages, dunes continentales, dunes côtières) à partir de la situation des nuages de points obtenus.

\subsection{Analyse en Composantes Principales (ACP)}

Cette méthode permet de mettre en évidence les ressemblances (corrélations) entre les différents paramètres granulométriques. Elle permet, en outre, de montrer la différence de comportement de groupes de sédiments vis-à-vis d'un ou plusieurs individus ou échantillons testés, définissant ainsi une zonation de la lagune en indiquant les évènements responsables de ce découpage (SORO, 2003).

Une Analyse en Composantes Principales (ACP) avec le logiciel Statistica sur l'ensemble des individus ou unités statistiques a été effectuée.

\section{Résultats et discussion}

\subsection{Morphologie de la lagune Adjin}

La figure 2 présente la carte bathymétrique de la lagune Adjin. Les profondeurs varient de 0 à $14 \mathrm{~m}$, mais elles peuvent atteindre $20 \mathrm{~m}$ à l'exutoire du fleuve Mé. Deux fonds lagunaires sont nettement identifiables (N'GUESSAN, 2008). Ce sont :

a) Le fond lagunaire principal de direction Ouest -Sud-Est, profond de 14 m maximum.

b) A l'Est le fond lagunaire situé dans le prolongement du canal naturel reliant la lagune Adjin à la lagune Potou.

Quelques hauts-fonds se rencontrent au Sud-Est à proximité de l'exutoire de la Mé, au Nord-Ouest, à proximité d'Anyama et aux débouchés de Djibi et Bété. Ils correspondraient au prolongement sous-lagunaire des alluvions de ces rivières (TASTET, 1979). Au Sud, non loin du village Adjin se localisent des hauts-fonds en nombre plus considérable. Ils ont une direction sensiblement parallèle à celle du canal naturel.

\subsection{Sédimentologie des sédiments superficiels de la lagune Adjin}

\subsubsection{Description lithologique des sédiments superficiels}

L'analyse macroscopique des sédiments superficiels de la lagune Adjin met en évidence quatre faciès lithologiques. Il s'agit de galets et graviers, de sables, de vases et de sédiments "mixtes" (N'GUESSAN et al., 2008) : 
a) Les galets et les graviers sont de couleur brun jaunâtre.

b) Les sables vont de très fins à grossiers. Leur couleur est brun jaunâtre. On y trouve, quelques débris végétaux et coquilliers.

c) Les vases sont de couleur variable, du noir olive au gris olive et avec une compacité variable. La vase organique d'aspect crémeux ou "crème" de vase a été identifiée (TASTET, 1979).

d) Les sédiments "mixtes" sont constitués de sables vaseux et de vases sableuses. Leur coloration varie du noir olive au gris olive. Ils contiennent des débris végétaux et coquilliers.

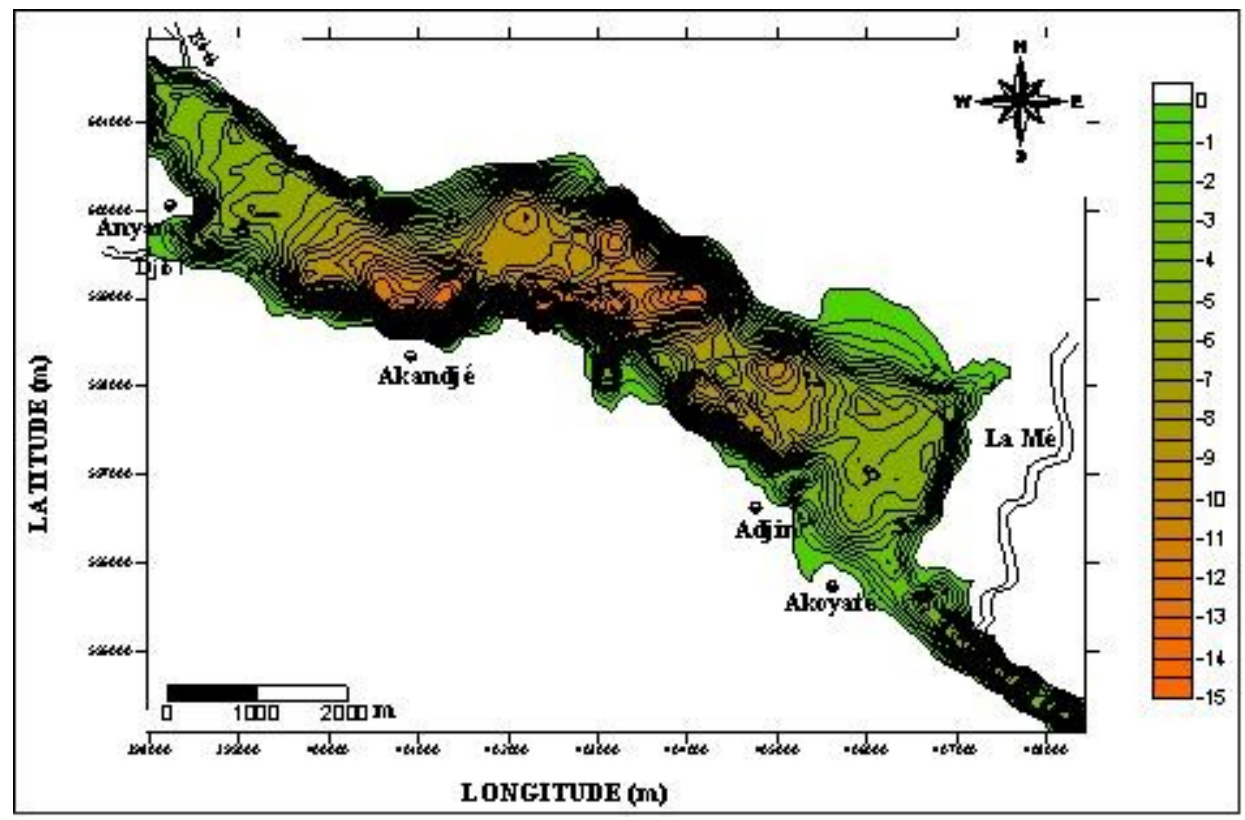

Figure 2. Carte bathymétrique de la lagune Adjin

\subsubsection{Répartition des faciès superficiels de la lagune Adjin}

La figure 3 présente la répartition des faciès sédimentaires de la lagune Adjin. Les galets et les graviers se localisent, au Nord et au Nord-Ouest de la lagune Adjin. Ces zones correspondent aux débouchés des rivières (Djibi et Bété) et à proximité de l'exutoire de la Mé. Ces sédiments se concentrent aux faibles profondeurs de l'ordre du mètre. Les sables occupent les berges de la lagune Adjin. Les sables proviennent des formations sableuses encaissantes soit par remaniement à partir des berges, soit par des apports de rivières. Ils se concentrent essentiellement à des profondeurs inférieures à $5 \mathrm{~m}$. Cela pourrait s'expliquer par le fait que les eaux de ruissellement qui transportent la plupart des sables atteignent une vitesse presque nulle au contact de la lagune. Le courant n'étant plus fort et donc incapable de transporter les grains de sables de taille plus importante, les déposes sur les berges à des profondeurs faibles. Les vases se rencontrent aux profondeurs supérieures à $5 \mathrm{~m}$. Elles se localisent dans les fonds 
lagunaires (voir figure 3). Cette accumulation est due au dépôt par décantation et au remaniement des zones de bordure par le clapot (TASTET \& GUIRAL, 1994). Les vases, de taille plus petite restent en suspension dans l'eau et se déposent par décantation à des profondeurs élevées (SANCHEZ \& LEVACHER, 2007).

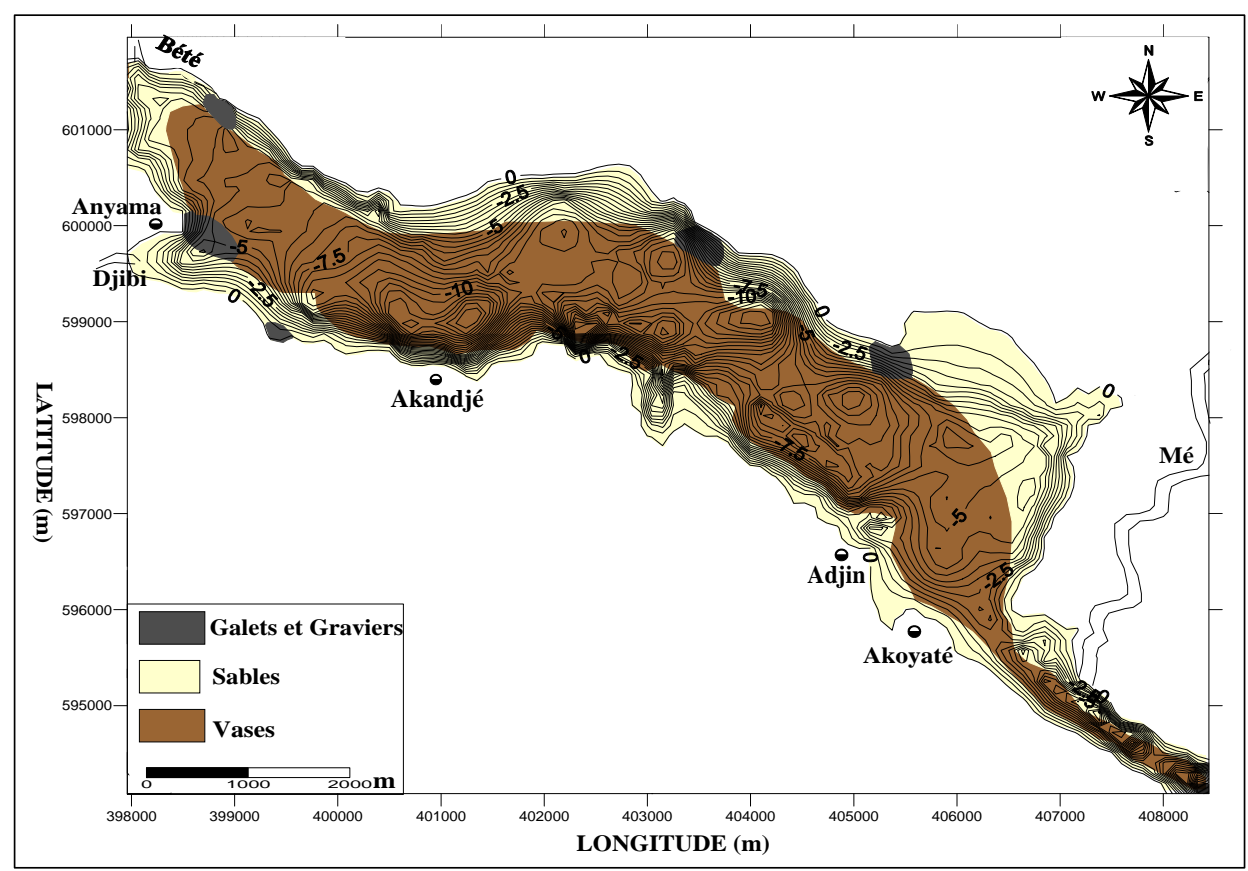

Figure 3. Répartition des faciès superficiels de la lagune Adjin.

\subsection{Mode de transport et environnement de dépôt des sédiments de la lagune Adjin}

\subsubsection{Mode de transport des sédiments}

Le test de VISHER (1969) réalisé sur les sédiments superficiels de la lagune Adjin donne les résultats présentés par la figure 4 . Il permet de montrer la relation entre la granulométrie des sédiments et leur mode de transport. Ainsi trois populations de sables se distinguent:

a) La population A regroupe les sables transportés par roulement.

b)La population B rassemble les sables transportés par saltation.

c) La population $\mathrm{C}$ est marquée par le transport des grains par suspension.

L'analyse du tableau 1, montre que la population B est la plus importante. Elle représente une proportion de $38 \%$. Les deux autres cas restant se repartissent entre la population C (33\%) et la population A (29\%). Cette analyse quantitative montre que la majorité des sédiments se déplace par saltation. Néanmoins, certains sédiments sont transportés par charriage et par suspension. 
Analyse granulométrique et environnement de dépôts des sables superficiels de la lagune Adjin (Côte d'Ivoire) : 6.7

Tableau 1. Proportion de sédiment selon les différents modes de transport.

\begin{tabular}{llll}
\hline & $\begin{array}{l}\text { Population A } \\
(0-10 \%)\end{array}$ & $\begin{array}{l}\text { Population B } \\
(10-90 \%)\end{array}$ & $\begin{array}{l}\text { Population C } \\
(90-100 \%)\end{array}$ \\
\hline Effectif par population & 70 & 90 & 80 \\
$\%$ par population & $29 \%$ & $38 \%$ & $33 \%$ \\
\hline
\end{tabular}

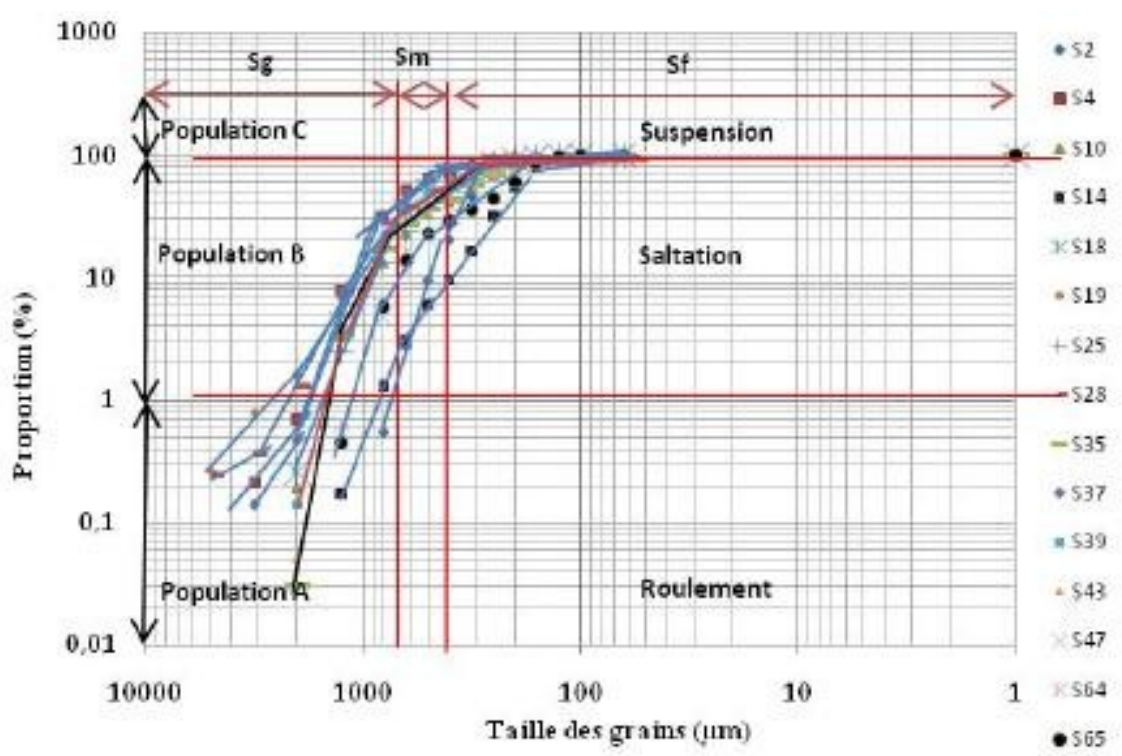

$\mathrm{Sg}$ : sables grossiers ; Sm : sables moyens ; Sf : sables fins

Figure 4. Test de Visher indiquant le mode de transport des sédiments en lagune Adjin.

Par ailleurs, la caractérisation du type de transport dans les différentes classes granulométriques représentée par le tableau 2 montre que :

a) Le mode de transport des sables fins $(\varnothing<0,315 \mathrm{~mm})$ est dominé par le transport par suspension ( $70 \%$ du matériel fin). Néanmoins 30\% de ces sables fins sont portés par saltation.

b)Les sables moyens de taille comprise entre 0,315 et $1,25 \mathrm{~mm}$ se déplacent essentiellement par saltation (93\% du matériel moyen). Eventuellement $7 \%$ de ces sables moyens bougent par charriage.

c) Le transport par charriage (ou roulement) est le mode de transport dominant des sables grossiers $(\varnothing>1,25 \mathrm{~mm})$ avec une proportion de $88 \%$ du matériel grossier. Par ailleurs $12 \%$ de ce matériel grossier est transporté par saltation.

Tableau 2. Caractérisation du type de transport selon les classes granulométriques.

\begin{tabular}{llll}
\hline & Roulement(\%) & Saltation(\%) & Suspension(\%) \\
\hline Sables grossiers & $88,16 \%$ & $11,84 \%$ & $0,00 \%$ \\
Sables moyens & $7,15 \%$ & $92,85 \%$ & $0,00 \%$ \\
Sables fins & $0,00 \%$ & $29,70 \%$ & $70,30 \%$ \\
\hline
\end{tabular}




\subsubsection{Environnement de dépôts des sédiments de la lagune Adjin}

Les diagrammes Md-So et Sk-Md de l'évolution des paramètres granulométriques des sables de la lagune Adjin permettent de déterminer leur milieu de dépôt. Le diagramme Md-So de la figure 5 présente la dispersion des points. Elle se fait entre le domaine de plages et le domaine de rivières. A l'analyse, tous les points sauf un seul sont regroupés dans le domaine des rivières. Ceci signifie que $96 \%$ des grains sont issus des rivières (voir tableau 3). Les sédiments pourraient dans ce cas être des sables d'origine fluviatile (MOIOLA \& WEISER, 1968). En effet ils seraient transportés par les rivières Djibi, Bété et le fleuve Mé avant d'être déposés en lagune. La majorité de ces sables provient des rivières Djibi, Bété et de la rive Nord de la lagune Adjin (voir figure 5). Toutefois, une quantité négligeable (4\%) des sables provient des plages. Ainsi, l'essentiel des sables de la lagune Adjin provient d'un environnement de type rivières.

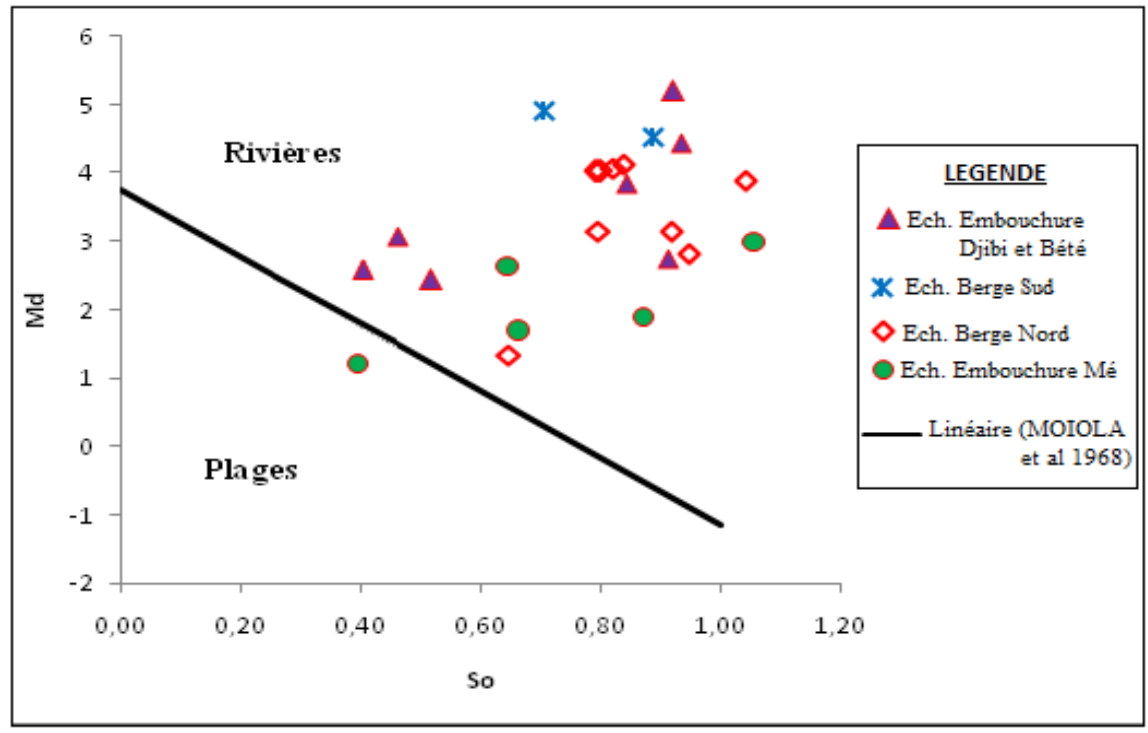

Figure 5. Diagramme Md-So (MOIOLA \& WEISER, 1968).

Tableau 3. Proportion de sédiment selon l'environnement de dépôt

(Diagramme Md-So).

\begin{tabular}{lll}
\hline & Rivières & Plages \\
\hline Effectif par environnement & 22 & 1 \\
$\%$ par environnement & $95,65 \%$ & $04,35 \%$ \\
\hline
\end{tabular}

La figure 6 présente le diagramme de dispersion Sk-Md. La répartition des points se fait dans le milieu des dunes continentales $(52 \%)$ et dans le milieu des dunes côtières $(48 \%)$. Ces proportions (voir tableau 4) ne permettent pas de caractériser l'origine de ces sables. Cela pourrait s'expliquer par le fait que la lagune Adjin joue un rôle d'interface entre le milieu continental et le milieu marin. La lagune est alimentée par un bassin 
Analyse granulométrique et environnement de dépôts des sables superficiels de la lagune Adjin (Côte d'Ivoire) : 6.9

principal qui draine les régions du socle précambrien (La Mé longue de $140 \mathrm{~km}$, avec un bassin versant de $4300 \mathrm{~km}^{2}$ (DURAND \& SKUBICH, 1982 ; PORT AUTONOME D'ABIDJAN, 2001)), auxquels s'ajoutent les apports des rivières Djibi et Bété (VARLET, 1978). Les sédiments pourraient être drainés des dunes côtières (48\%) par les rivières et par les eaux de ruissellement avant d'être déposés en lagune. Toutefois, le domaine des dunes continentales serait l'environnement de dépôt des sables de la lagune Adjin (MOIOLA \& WEISER, 1968).

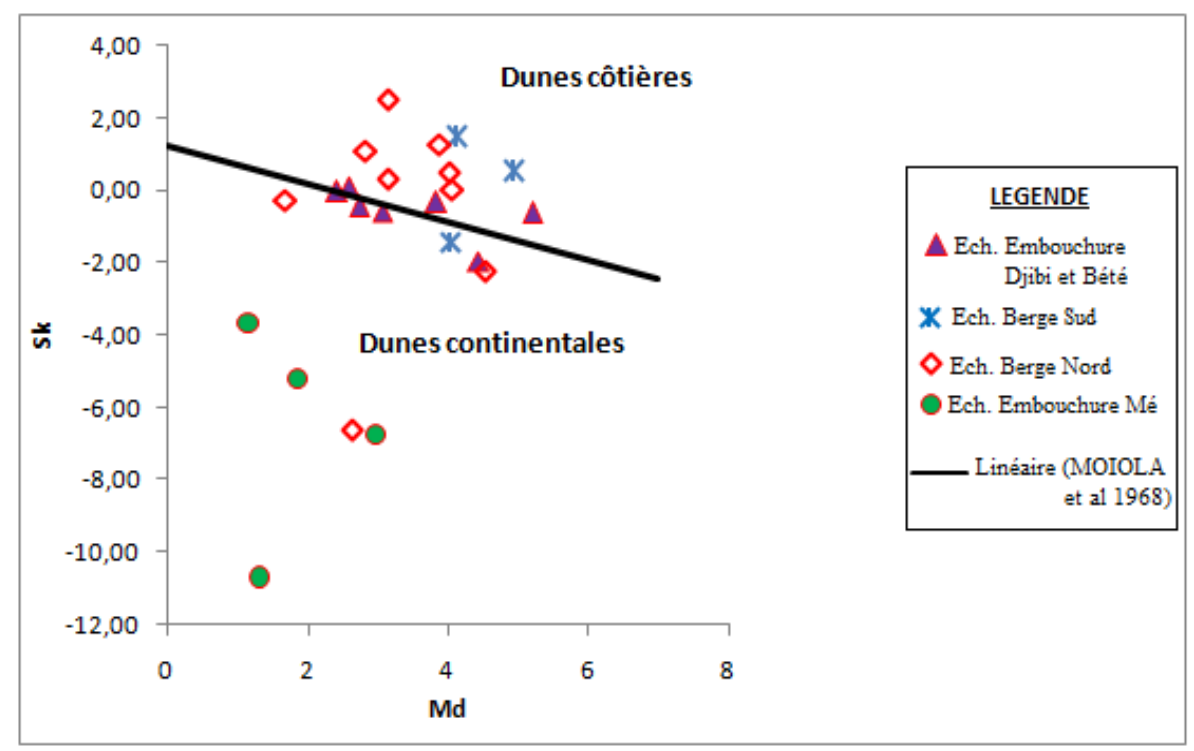

Figure 6. Diagramme Sk-Md (MOIOLA \& WEISER, 1968).

Tableau 4. Proportion de sédiment selon l'environnement de dépôt (Diagramme Sk-Md).

\begin{tabular}{lll}
\hline & Dunes côtières & Dunes continentales \\
\hline Effectif par environnement & 11 & 12 \\
$\%$ par environnement & $47,83 \%$ & $52,17 \%$ \\
\hline
\end{tabular}

3.4 Analyses en Composantes Principales des paramètres granulométriques des sables de la lagune Adjin

La matrice de corrélation représentée par le tableau 5 montre les relations entre les paramètres granulométriques des sédiments pris deux à deux. Son analyse montre qu'il existe une très bonne corrélation $(\mathrm{r}=0,97)$ entre la Médiane $(\mathrm{Md})$ et la Moyenne $(\mathrm{M})$; la Médiane et le Mode (Mo); le Mode et la Moyenne. La corrélation est moyenne $(\mathrm{r}=0,52)$ entre le Skewness (Sk) et M, le Sorting (So) et M. Une anticorrélation $(r=-0,60)$ entre l'Indice de Triage et les autres paramètres granulométriques étudiés a été déterminée (Md, So, Mo, M et Sk). 
Ces différentes corrélations révèlent les interactions entre la Médiane, la Moyenne, le Mode. Il existe aussi des relations entre la Moyenne, le Skewness et le Sorting. Ces interdépendances s'expliqueraient soit par une origine commune (ou même plan factoriel) soit par un processus identique régulant leur évolution dans l'environnement. La valeur négative du coefficient de corrélation entre IT et les autres paramètres, traduit des interactions inverses. Ces paramètres granulométriques n'ont ni le même plan factoriel ni le même mécanisme déterminant leur évolution dans le milieu.

Tableau 5. Matrice de corrélation.

\begin{tabular}{lllllll}
\hline & Sk & Md & So & Mo & IT & M \\
\hline Sk & 1,000000 & & & & & \\
Md & 0,471705 & 1,000000 & & & & \\
So & 0,098023 & 0,484355 & 1,000000 & & & \\
Mo & 0,320980 & $\mathbf{0 , 8 9 8 4 0 7}$ & 0,472546 & 1,000000 & & \\
$\boldsymbol{I T}$ & $\mathbf{- 0 , 3 2 1 3 7 3}$ & $\mathbf{- 0 , 5 9 8 7 1 9}$ & $\mathbf{- 0 , 9 4 1 7 5 5}$ & $\mathbf{- 0 , 5 3 4 2 3 7}$ & 1,000000 & \\
$\boldsymbol{M}$ & 0,523871 & $\mathbf{0 , 9 7 1 3 1 1}$ & 0,580608 & $\mathbf{0 , 8 4 2 8 3 0}$ & $\mathbf{- 0 , 7 2 3 5 1 1}$ & 1,000000 \\
\hline
\end{tabular}

Le tableau des valeurs propres montre que les deux premiers axes factoriels expriment respectivement $67,39 \%$ (Fact. 1) et 18,25\% (Fact. 2) de la variance exprimée. Ces facteurs fournissent à eux seuls $85,64 \%$ de l'information statistique. L'Analyses en Composantes Principales (ACP) ne tiendra compte que de ces trois facteurs pour l'analyse des plans factoriels. Cette approche permettra d'apprécier l'évolution des différents paramètres granulométriques en fonction des axes factoriels Fact.1 et Fact. 2.

Tableau 6. Valeurs propres.

\begin{tabular}{lllll}
\hline & Val. Propre & \% Total & Cumul (Val. P) & Cumul (\%) \\
\hline $\mathbf{1}$ & 4,043226 & 67,38710 & 4,043226 & $\mathbf{6 7 , 3 8 7 1}$ \\
$\mathbf{2}$ & 1,095112 & 18,25187 & 5,138339 & $\mathbf{8 5 , 6 3 9 0}$ \\
$\mathbf{3}$ & 0,693601 & 11,56002 & 5,831940 & 97,1990 \\
$\mathbf{4}$ & 0,130476 & 2,17461 & 5,962416 & 99,3736 \\
$\mathbf{5}$ & 0,032008 & 0,53347 & 5,994424 & 99,9071 \\
$\mathbf{6}$ & 0,005576 & 0,09293 & 6,000000 & 100,0000 \\
\hline
\end{tabular}

\subsection{Plan factoriel Fact 1- Fact 2}

Le plan factoriel Fact. 1 est bipolaire et regroupe plus de $67 \%$ des informations. Son pôle positif est défini par Mo, M, Md et à son pôle négatif IT. IT évolue de façon contraire par rapport à Mo, M et Md fortements corrélés. Le plan factoriel Fact. 2, bipolaire extrait plus de $18 \%$ des informations. Le côté positif est marqué par So, et le côté négatif par Sk (voir figure 7). Les coefficients de corrélation entre ces paramètres 
granulométriques admettent cette hypothèse. Ils sont soumis à un même phénomène et ont une origine commune (voir figure 7).

La bonne corrélation entre la Médiane, la Moyenne et le Mode, avec des coefficients de corrélations voisins de 1 , indique une forte proportionnalité entre ces paramètres granulométriques. Ainsi, le grain moyen des sables superficiels de la lagune Adjin appartient à la classe granulométrique la plus importante et avec une taille des particules correspondant à un pourcentage cumulé de 50\%. Ces trois paramètres sont influencés par la vitesse du courant de transport. IT est aussi influencé par la vitesse du courant de transport et évolue inversement par rapport aux autres paramètres granulométriques.

So et Sk sont influencés par le poids (densité) des grains de sable de la lagune Adjin, mais évoluent inversement entre eux. Ainsi, le classement des grains de sables en lagune Adjin est lié à la prépondérance d'éléments fins ou grossiers dans un sédiment. Ils évoluent inversement et sont influencés par le poids des sédiments.

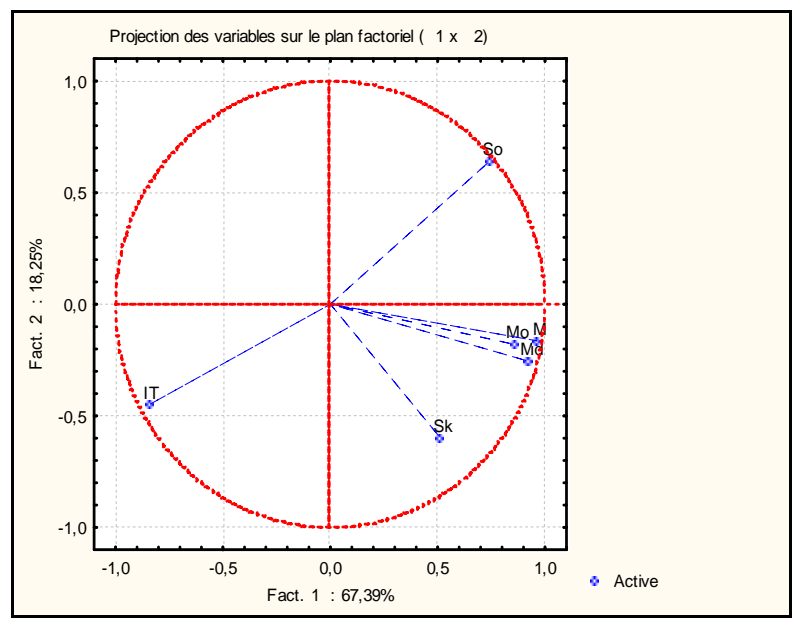

Figure 7. Espace des variables du plan factoriel Fact.1 X Fact.2 Espace des unités statistiques

On distingue deux familles F1 et F2 (voir figure 8). La première famille (F1) d'unité statistique regroupent les points $(1,2,4,7,19-21)$. Ces échantillons proviennent des débouchés de la Mé et des rivières Djibi et Bété en lagune Adjin. Le courant de transport est donc plus fort dans ce cas. Ceci montre que les paramètres granulométriques sont influencés par la vitesse du courant de transport des sédiments. La famille 2 (F2) rassemble les points (3, 5, 6, 8-18, 22, 23). Les échantillons regroupés dans cette famille se localisent sur les berges Nord et Sud et dans les chenaux lagunaires. Les eaux de ruissellement déposent les sédiments en lagune par un courant plus faible. Dans les fonds lagunaires le courant est faible, avec un environnement de dépôt plus calme. Ceci pourrait expliquer l'influence des paramètres granulométriques par la vitesse du courant et le poids des sables qu'il transporte. 


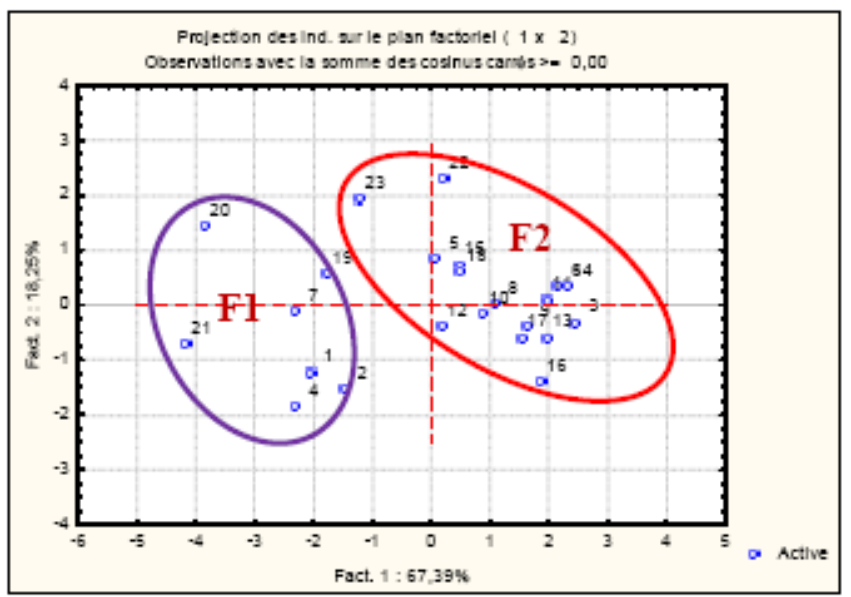

Figure 8. Espace des unités statistiques dans le plan Fact. 1-Fact. 2.

\section{Conclusions}

La lagune Adjin est caractérisée par des profondeurs de 0 à $14 \mathrm{~m}$ pouvant atteindre $20 \mathrm{~m}$ à l'exutoire de la Mé. Un fond lagunaire principal, profond de $14 \mathrm{~m}$ maximum et à l'Est un chenal situé dans le prolongement du canal naturel reliant la lagune Adjin à la lagune Potou se distingue. Les hauts-fonds qui s'y rencontrent se localisent au Nord-Ouest, au Sud et au Sud-Est de la lagune Adjin.

L'étude lithologique et granulométrique des sédiments superficiels, indique que les galets et les graviers, se localisent par endroit, sur le pourtour de la lagune à des profondeurs de l'ordre du mètre, et à proximité des débouchés des rivières Djibi et Bété. Les sables occupent la bordure de la lagune à des profondeurs inférieures à 5 mètres. Les vases se concentrent dans les chenaux à des profondeurs supérieures à 5 mètres.

L'étude de l'environnement de dépôts nous amène à dire que, l'essentiel des sables de la lagune Adjin provient d'un environnement de type rivières. En effet ils sont transportés par les rivières Djibi, Bété et le fleuve Mé avant d'être déposés en lagune. La majorité de ces sables proviennent des rivières Djibi, Bété et de la rive Nord de la lagune Adjin. Les sédiments pourraient être drainés des dunes côtières (48\%) par les rivières et par les eaux de ruissellement avant d'être déposés en lagune. Toutefois, le domaine des dunes continentales serait l'environnement de dépôt des sables de la lagune Adjin.

L'analyse quantitative montre que la majorité des sédiments se déplace par saltation. Toutefois, certains sédiments se déplacent par charriage et par suspension. Le mode de transport des sables fins est dominé par le transport par suspension $(70 \%$ du matériel fin). Les sables moyens se déplacent essentiellement par saltation $(93 \%$ du matériel moyen). Le transport par roulement est le mode de transport dominant des sables grossiers avec une proportion de $88 \%$ du matériel grossier. 
Analyse granulométrique et environnement de dépôts des sables superficiels de la lagune Adjin (Côte d'Ivoire) : 6.13

\section{Références bibliographiques}

ABE J. (2005). Contribution à la connaissance de la morphologie et de la dynamique du littoral ivoirien (cas du littoral d'Abidjan). Essais de modélisation en vue d'une gestion rationnelle. Thèse Doctorat d'Etat, Université Cocody, Abidjan, Côte d'Ivoire, $345 \mathrm{p}$.

BOUMIR K. (1985). Les sables fauves de la région de Méknès : éléments d'analyse sédimentologique. Bulletin de l'institut scientifique, Rabat, ${ }^{\circ}$ 9, pp 107-116.

DURAND J.R., SKUBICH M. (1982). Les lagunes ivoiriennes. Aquaculture, 27, pp 211-250. doi:10.1016/0044-8486(82)90059-X

FOLK R.L., WARD W.C. (1957). Brazos river bar, a study in the significance of grain size parameters. Journal of Sedimentary Petrology, $\mathrm{n}^{\circ} 27$, pp 3- 26.

LOWRANCE (1998). LMS-160 et Global Map 1600 ; Directive d'installation et note technique. Lowrance Electronics, Inc. 75 p.

MOIOLA R.J., WEISER D. (1968). Textural parameters: an evaluation. Journal of Sedimentary Petrology, $\mathrm{n}^{\circ} 38$, pp 45- 53. doi:10.1306/74D718C5-2B21-11D7-8648000102C1865D

N'GUESSAN Y.A. (2008). Analyse morphologique, sédimentologique et environnement de dépôts des sédiments superficiels des lagunes Adjin et Potou (Zone littorale de la Côte d'Ivoire). Thèse Unique, Université Cocody, Abidjan, Côte d'Ivoire $171 \mathrm{p}$.

N'GUESSAN Y.A., MONDE S., AKA K. (2008). Sedimentological analysis and paleoenvironement of the surface sediments of the Adjin and Potou lagoons. European Journal of Scientific Research, Vol. 19, n² 2, pp 391- 404.

PORT AUTONOME D'ABIDJAN (2001). Etude sur modèle physique sédimentologique pour une ouverture permanente de l'embouchure de la Comoé. Rapport général. Rapport 552014 R2. SOGREAH, 45 p.

SANCHEZ M., LEVACHER D. (2007). The influence of particle size of the dispersed mineral fraction on the settlement of marine and estuarine muds. Geo-Marine Letters. Vol. 27, $\mathrm{n}^{\circ}$ 5, pp 303-313. doi:10.1007/s00367-007-0053-7

SORO G. (2003). Evaluation des métaux lourds (Co, Cr, Mn, Ni et $\mathrm{Zn}$ ) dans les sédiments de la lagune Ebrié, Abidjan (Côte d'Ivoire). Mémoire de DEA, Univ. Cocody, Côte d'Ivoire, 70 p.

TASTET J.P. (1979). Environnements sédimentaires et structuraux quaternaires du littoral du golfe de Guinée (Côte d'Ivoire, Togo et Bénin). Thèse Doctorat d'Etat, Université Bordeaux I, France, 175 p.

TASTET J.P., GUIRAL D. (1994). Géologie et sédimentologie. in : Environnement et ressources aquatiques de Côte d'Ivoire, t. II. Les milieux lagunaires, édition ORSTOM, pp 35- 58.

VARLET F. (1978). Le régime de la lagune Ebrié (Côte d'Ivoire), Traits physiques essentiels. Travaux et Documents ORSTOM, n 83, 110 figs., 164 p. 
6.14 : Revue Paralia - Vol. 4 (2011)

VISHER G.S. (1969). Grain size distributions and depositional processes. Journal of Sedimentary Petrology, Vol. 39, $\mathrm{n}^{\circ}$ 3, pp 1074-1106. doi:10.1306/74D71D9D-2B21-11D7$8648000102 \mathrm{C} 1865 \mathrm{D}$

WOGNIN A.V.I. (2004). Hydrologie et sédimentologie à l'embouchure du fleuve Bandama. Thèse de Doctorat, Université Cocody, Abidjan, Côte d'Ivoire, 195 p. 